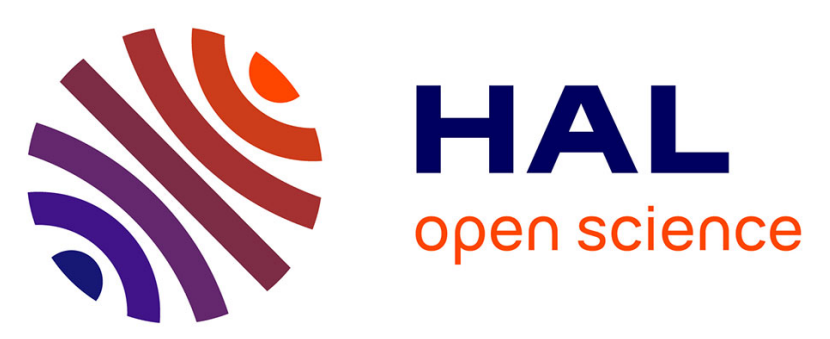

\title{
A model for transfer of P-glycoproteins in MCF-7 breast cancer cell line with multiple transfer rules
}

Pierre Magal, Ahmed Noussair, Jennifer Pasquier, Pascal Zongo, Frank Le Foll

\section{To cite this version:}

Pierre Magal, Ahmed Noussair, Jennifer Pasquier, Pascal Zongo, Frank Le Foll. A model for transfer of P-glycoproteins in MCF-7 breast cancer cell line with multiple transfer rules. Bulletin of Mathematical Biology, 2017, 79 (9), pp.2049 - 2067. 10.1007/s11538-017-0319-9 . hal-01683780

\section{HAL Id: hal-01683780 https://hal.univ-antilles.fr/hal-01683780}

Submitted on 14 Jan 2018

HAL is a multi-disciplinary open access archive for the deposit and dissemination of scientific research documents, whether they are published or not. The documents may come from teaching and research institutions in France or abroad, or from public or private research centers.
L'archive ouverte pluridisciplinaire HAL, est destinée au dépôt et à la diffusion de documents scientifiques de niveau recherche, publiés ou non, émanant des établissements d'enseignement et de recherche français ou étrangers, des laboratoires publics ou privés. 


\title{
A model for transfer of P-glycoproteins in MCF-7 breast cancer cell line with multiple transfer rules
}

\author{
P. Magal, A. Noussair \\ Univ. Bordeaux, IMB, UMR 5251, F-33400 Talence, France. \\ CNRS, IMB, UMR 5251, F-33400 Talence, France. \\ J. PASQUieR \\ Stem Cell and Microenvironment Laboratory, Weill Cornell Medical College \\ in Qatar, Education City, Qatar Foundation, Doha PO: 24144, Qatar \\ Department of Genetic Medicine, Weill Cornell Medical College, New York, NY, USA \\ P. ZONGO \\ Université des Antilles, DSI and IUT B.P. 7209, 97275 Schoelcher cedex, France \\ F. LE FOLL \\ Laboratory of Ecotoxicology, University of Le Havre, 76058 Le Havre, France
}

June 8, 2017

\begin{abstract}
In this paper, we consider a direct protein transfer process between cells in co-culture. Assuming that cells continually encounter each other, and from some hypotheses on cell-to-cell rules of transfer, we derive discrete and continuous Boltzmann-like integrodifferential equations. The novelty of this model is to take into account multiple transfer rules. This new transfer model is used to fit the experimental data of cell-to-cell protein transfer in breast cancer.
\end{abstract}

Keywords: Breast cancer cells, protein transfers, discrete and continuous models.

\section{Introduction}

The objective of this paper is to analyse a population dynamics model for a distribution of cells with respect to a quantity of protein called P-glycoprotein (P-gp). We consider a co-culture of two variants of human breast cancer cell 5 lines, the wild-type chemotherapy sensitive MCF-7 cells which are sensitive to chemotherapy, and the MCF-7/Doxo cells which are resistant to cytotoxic treatments by P-gp overexpression. We refer to Pasquier et al. 14 for more information about the biological background. We assume that all cells continually encounter each other. Each pairwise encounter results a direct cell-to-cell 
protein transfer. We focus particularly on the size content of the transferable matter with the conservation of the total transferred mass and of the total population.

In this article, we examine different strategies of exchange of P-pg with different efficient transfer rates. We investigate how to model some new transfer 15 rules in systems constituted by a large number of interacting cells. Actually the main novelty compared to the previous existing models is to allow two different types of transfer rate. This corresponds to the MCF-7 and MCF-7/Doxo ability to transfer exchange P-gp by direct contact.

Recent studies have shown that cells can communicate by the transfer of membrane proteins 6]. For instance, the direct transfer of P-gp between cells was studied in 1, 12, 16, 14, 15. Because P-gp is acting as a drug-efflux pump, its transfer may confer resistance against cytotoxic drugs to the recipient cells. Another example is the transfer of human immunodeficiency virus (HIV) from an infected cell to an uninfected cell 5. Moreover it has been shown in recent

25 studies that the $\alpha$-synuclein can be intercellularly transferred, which could be a key element in the spread of Parkinson disease pathology [10].

Mathematical models have already been proposed to study the transfer of physical quantities between particles with Boltzmann-type equation (see review in [17). These types of models have also been used in population dynamics

зо [2, 3, 4]. Recently an integro-partial differential Boltzmann-type equation for cell population dynamics have been analysed in [11. Furthermore, this model was used to analyse the consequences of cell-to-cell P-glycoprotein transfer on acquired multidrug resistance in breast cancer [14. In [13, a stochastic model was developed to study the horizontal transfer of genes in microbial popula-

35 tions. The goal of this paper is to provide a new class of mathematical models which include different strategies of transfer rates and direct cell-to-cell rules of transfers, which can explain experimental data.

In section 2, we summarize some basic assumptions of the experimentation. We then discuss, in section 3, the transfer rules and derive a new mathematical 40 model. A discrete model is presented in section 4 . In sections 5 and 6 we compare the model to the experimental data. We will also provide in the appendix a result of convergence of the discrete model to the continuous model.

\section{Methods and basic assumptions}

In 14, we have shown that MCF-7 cells acquired resistance to chemotherapeutic treatments by direct transfer of a protein called P-glycoprotein (P-gp) from resistant cells to sensitive cells in vitro. Figure 1) shows the protein transfer between these cells in co-culture. The red cells correspond to resistant cells, and the green cells correspond to sensitive cells. Our goal, in this paper, is to describe the resistance acquired by sensitive cells from resistant cells by such a so cell-to-cell direct transfer. 

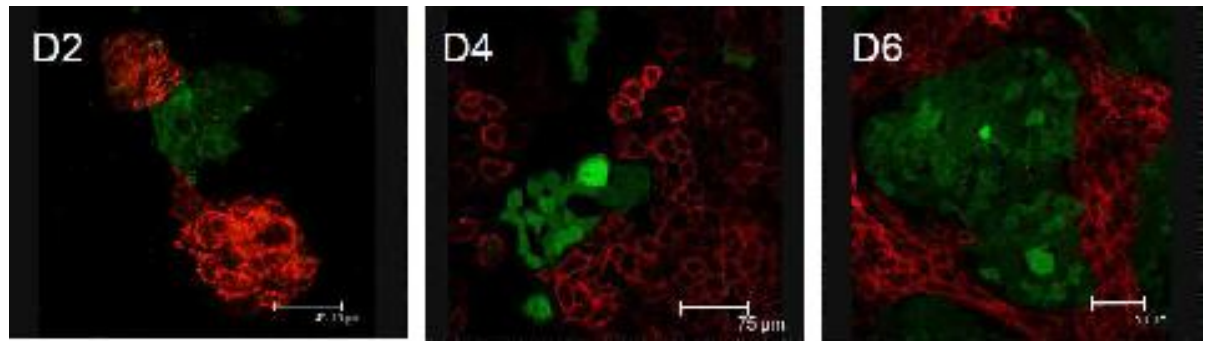

Figure 1: (Color online) Confocal imaging of cell-to-cell interactions in the human breast cancer cell line, MCF-7. Wild-type sensitive MCF-7 were cytosol-tagged with the persistent probe CellTracker Green (green fluorescence, ctgMCF-7) P-glycoprotein (P-gp) overexpressing chemotherapy-resistant cells were membrane-tagged with a phycoerythrin-conjugate antibody directed against a P-Pgp (red fluuorescence, peMCF-7). A mixture of $50: 50$ of ctgMCF-7 and peMCF-7 was co-cultured over six days. Images were obtained by confocal laser scanning microscopy. Photos were taken at day 2 (D2) (scale bar:15 $\mu \mathrm{m}$ ), day 4 (D4) (scale bar: $75 \mu \mathrm{m}$ ), and day 6 (D6) (scale bar:80 $\mu \mathrm{m})$. Note that cells become organized into well-delimited islets over the 6-day time course. This figure illustrates the experimental conditions.

For simplicity, in the schematic and in the model, we assume that the transferable matter is transferred from a donor population to a second recipient population. We assume that cells are cultured as adherent cells in a dish. The cell can exchange proteins or other molecules with cells around it. We assume 55 that when a contact between cells occurs, the donor cell can donate a small portion of the proteins to the recipient cell. This process may actually occur as exchange of protein portions, but here we describe only the direct transfer of observable proteins present exclusively in the donor cells.

Experimental procedures: In previous studies [14, 15], we have demonstrated that intercellular transfers of functional P-glycoprotein occur through donor-recipient interactions in the absence of drug selection pressure in MCF7 human breast cancer cell line. Briefly, in these experiments, drug-sensitive parental MCF-7 cells were tagged with the persistent fluorescent probe cytotracker green, mixed 50:50 and co-cultured with P-gp overexpressing drugresistant variants, in the absence of treatment. Transfers of P-gp were monitored over 7 days in 50:50 co-cultures by immunodetection of the protein using confocal microscopy, as illustrated in Figure 1 (parental MCF-7 in green, Pgp in red). Parental and drug-sensitive MCF-7 were of similar growth rates, 0.6195 and 0.6328 respectively [14]. Co-cultures were interrupted before cell

70 confluency. Transwell co-incubation experiments, where cells were physically separated signaling-molecules permeable porous membranes, proved the noninfluence of local soluble factors capable of gene induction in the acquired resistance $[15]$.

P-gp expression levels in cell membranes as well as drug efflux capacities were quantitatively followed over time by flow cytometry. This latter phenotype, 
leading to drug resistance, was used to generate the set of experimental data used in the present study. As depicted in Figure 3, parental MCF-7 with no efflux activity accumulated the fluorescent probe calcein-AM and were distributed in the right side of the histogram (region R2), while probe-expelling cells populated the left part of the graph (region R1).

Our goal, in this paper, is to describe the resistance acquired by sensitive cells from resistant cells by cell-to-cell direct transfer.

\section{Derivation of a continuous model describing transfers}

85 Consider a population of cells in a co-culture, where each cell possesses an amount of protein to be partially transferred due to some specific rules. Assume that cells continually encounter other cells. Each pairwise of encounter during the transfer time results a winner "Recipient cell" and a loser "Donor cell" or a loser "Recipient cell" and a winner "Donor cell". Then in order to fully

90 determine the transfer rules we will use two types of (deterministic type) transfers when the transfer occurs between two given cells. The basic assumptions used to describe the transfers are the following.

Assumption 1 (i) The probability that a pair of two individuals are involved in a transfer event is independent of their $p$ values and the pairing is chosen randomly from all individuals.

(ii) The time between two transfer events follows an exponential law with mean $\tau^{-1}>0$ (alternatively, $\tau$ is the rate of transfer per unit time).

(iii) Let $f \in L^{\infty}(\mathbb{R}, \mathbb{R})$ be an even function with $0 \leq f \leq 1$ (we call $f$ the transfer efficiency). If two individuals whose difference in quantity is $p$ are involved in a transfer, then the one with higher value loses $f(p)$ times the difference of their $p$ values and the one with lower $p$ value gains exactly this amount.

Recall that with the above assumption a transfer model was proposed by Hinow et al. 11]. Actually Assumption 1 corresponds precisely to the following differential equation

$$
\left\{\begin{array}{l}
\frac{\partial u(t, x)}{\partial t}=2 \tau\left[T_{0}(u(t, .))(x)-u(t, x)\right], \text { for } x \in \mathbb{R}, \\
u(0, .)=u_{0} \in L_{+}^{1}(\mathbb{R}) .
\end{array}\right.
$$

In this model $u(t, x)$ is the density of cells, that is to say that

$$
\int_{x_{1}}^{x_{2}} u(t, x) d x
$$

is the number of cells with transferable quantity $x \in\left(x_{1}, x_{2}\right)$. Assumption 1 1 (ii) means that the flux of cells that transferring is $2 \tau \int_{\mathbb{R}} u(t, x)$ at a given time 
$t$. This corresponds to the term $-2 \tau u(t, x)$ in system (3.1). The distribution obtain after one transfer (this flux of transferring cells) is given by the transfer operator $2 \tau T_{0}(u)(x)$. The operator of transfer is defined by

$$
T_{0}(u)(x):=\left\{\begin{array}{l}
\frac{\widehat{T}_{0}(u)(x)}{\int_{\mathbb{R}} u(r) d r} \text { if } \int_{\mathbb{R}} u(r) d r \neq 0, \\
0 \text { otherwise },
\end{array}\right.
$$

where

$$
\widehat{T}_{0}(u)(x):=\int_{\mathbb{R}} u(x+f(p) p) u(x-(1-f(p)) p) d p .
$$

In this article we will extend Assumption 1 1 (iii) by considering the case of two transfer efficiencies. For a given transfer between two cells, we now choose randomly the transfer efficiency (in between two function $f_{1}$ and $f_{2}$ ). Ones the transfer efficiency is fixed, we use the same idea than in model (3.1) to describe the transfers.

Assumption 2 Let $f_{1}, f_{2} \in L^{\infty}(\mathbb{R})$ be two even functions with $0 \leq f_{1} \leq 1 / 2$ and $0 \leq f_{2} \leq 1 / 2$ (two transfer efficiencies). If two individuals whose difference in quantity is $p$ are involved in a transfer, then the one with higher value loses $f_{1}(p)$ (respectively $f_{2}(p)$ ) times the difference of their $p$ values and the one with lower $p$ value gains exactly this amount with the probability $\pi_{1}(p)$ (respectively with the probability $\pi_{2}(p)$ ).

More precisely we will make the following assumption

Assumption 3 We assume that $0 \leq\left(f_{1}+f_{2}\right)(p)<1$ for almost every $p \in \mathbb{R}$.

Then we will define the probabilities $\pi_{1}(p)$ and $\pi_{2}(p)$ by

$$
\pi_{1}(p):=\frac{\left[\frac{1}{2}-f_{2}(p)\right]}{\left[1-\left(f_{1}+f_{2}\right)(p)\right]} \text { and } \pi_{2}(p):=\frac{\left[\frac{1}{2}-f_{1}(p)\right]}{\left[1-\left(f_{1}+f_{2}\right)(p)\right]} .
$$

One may observe that

$$
\pi_{1}(p)+\pi_{2}(p) \equiv 1
$$


(a)

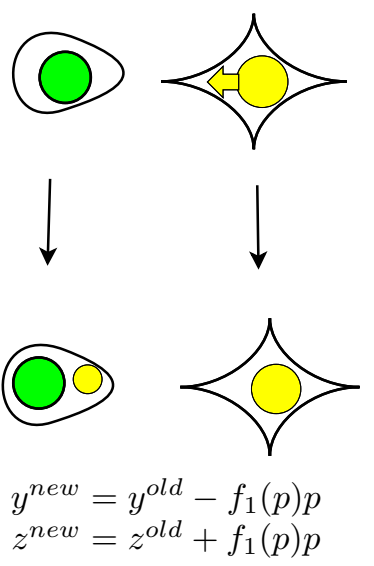

(c)

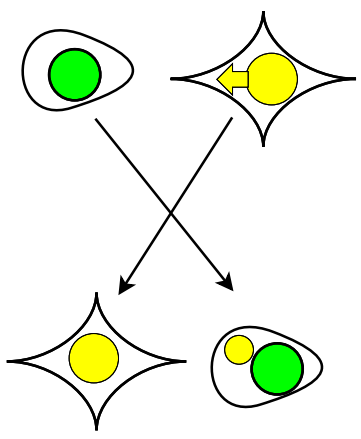

$$
\begin{aligned}
& y^{\text {new }}=z^{\text {old }}+f_{1}(p) p \\
& z^{\text {new }}=y^{\text {old }}-f_{1}(p) p
\end{aligned}
$$

(b)
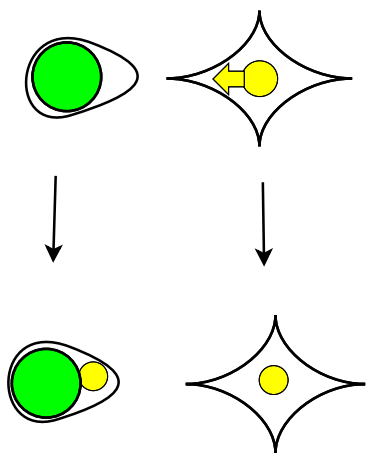

$y^{\text {new }}=y^{\text {old }}-f_{2}(p) p$ $z^{\text {new }}=z^{\text {old }}+f_{2}(p) p$

(d)

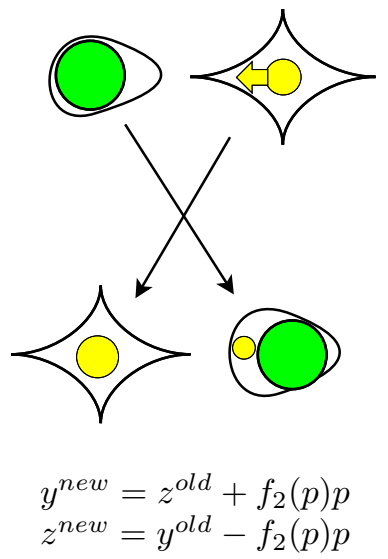

Figure 2: This figure illustrates the rule of transfer Assumption 2. We assume that the quantities of transferable quantities for each cells are $y^{\text {old }}$ and $z^{\text {old }}$ (respectively $y^{\text {new }}$ and $z^{\text {new }}$ ) before transfer (respectively after transfer). In the above figure $p:=y^{\text {old }}-z^{\text {old }}$ is assumed to be positive. The figures (a) and (c) correspond to the fraction transferred is $f_{1}(p)$ while the figures (b) and (d) correspond to the transfer efficiency $f_{2}(p)$. As explained in Assumption 2 the transfer efficiency $f_{1}(p)$ is used with a probability $\pi_{1}(p)$ while the transfer efficiency $f_{2}(p)$ is used with a probability $\pi_{2}(p)$.

Remark 4 In order to compare the model with real data, we will assume that $f_{1}$ and $f_{2}$ are two step functions (see (5.2)-(5.3)). More precisely, we will assume 
that there exist two positive real numbers $\delta_{\max }>\delta_{\min }>0$ such that for each $i=1,2$

$$
f_{i}(p):=\left\{\begin{array}{l}
\bar{f}_{i} \text { if } p \in\left(\delta_{\min }, \delta_{\max }\right) \\
\epsilon \bar{f}_{i} \text { otherwise. }
\end{array}\right.
$$

with $f_{i}>0$ and $\epsilon \in[0,1]$ (very small). This mean that exchanges takes place 120 mostly if the difference of the transfer pair $p \in\left(\delta_{\min }, \delta_{\max }\right)$. Otherwise if $p \notin$ $\left(\delta_{\min }, \delta_{\max }\right)$ the fraction transferred is very small. Therefore the quantities $\delta_{\min }$ and $\delta_{\max }$ can be regarded as a transfer threshold.

Under the above assumptions the model describing the transfers is the following

$$
\left\{\begin{array}{l}
\frac{\partial u(t, x)}{\partial t}=2 \tau[T(u(t, .))(x)-u(t, x)], \text { for } x \in \mathbb{R} \\
u(0, .)=u_{0} \in L_{+}^{1}(\mathbb{R}) .
\end{array}\right.
$$

The operator describing the rule for one transfer is defined by

$$
T(u)(x)=\left\{\begin{array}{l}
\frac{\widehat{T}(u)(x)}{\int_{\mathbb{R}} u(r) d r} \text { if } \int_{\mathbb{R}} u(r) d r \neq 0, \\
0 \text { otherwise, }
\end{array}\right.
$$

where

$$
\begin{aligned}
\widehat{T}(u)(x) & :=\int_{\mathbb{R}} \pi_{1}(p) u\left(x+f_{1}(p) p\right) u\left(x-\left(1-f_{1}(p)\right) p\right) d p \\
& +\int_{\mathbb{R}} \pi_{2}(p) u\left(x+f_{2}(p) p\right) u\left(x-\left(1-f_{2}(p)\right) p\right) d p
\end{aligned}
$$

125 The preservation of the total number of cells, and the preservation of the total mass of transferable quantity follow from the following equalities.

Lemma 5 Let Assumptions 2 and 3 be satisfied. We have the following properties

(i) (Preservation of the total number of cells) For each $u \in L_{+}^{1}(\mathbb{R})$ we have $T(u) \in L_{+}^{1}(\mathbb{R})$ and

$$
\int_{\mathbb{R}} T(u)(x) d x=\int_{\mathbb{R}} u(x) d x
$$

(ii) (Preservation of the total mass of transferable quantities) For each $u \in L_{+}^{1}(\mathbb{R})$ such that $\int_{\mathbb{R}} x u(x) d x<+\infty$ we have

$$
\int_{\mathbb{R}} x T(u)(x) d x=\int_{\mathbb{R}} x u(x) d x
$$


Remark 6 The transfer operator $T$ also preserves the support of the distribution $u$. Actually if $u$ is only defined on a subinterval $I \subset \mathbb{R}$ then we will define $\widehat{T}$ as

$$
\begin{aligned}
\widehat{T}(u)(x) & :=\int_{\mathbb{R}} \pi_{1}(p) \bar{u}\left(x+f_{1}(p) p\right) \bar{u}\left(x-\left(1-f_{1}(p)\right) p\right) d p \\
& +\int_{\mathbb{R}} \pi_{2}(p) \bar{u}\left(x+f_{2}(p) p\right) \bar{u}\left(x-\left(1-f_{2}(p)\right) p\right) d p
\end{aligned}
$$

where

$$
\bar{u}(x):=\left\{\begin{array}{l}
u(x) \text { if } x \in I \\
0 \text { otherwise }
\end{array}\right.
$$

Proof. Consider

$$
V:=\int_{\mathbb{R}} u(r) d r \int_{\mathbb{R}} \phi(x)[T(u)(x)-u(x)] d x .
$$

We have

$$
\begin{aligned}
V & =\int_{\mathbb{R}} \int_{\mathbb{R}} \pi_{1}(p) \phi(x) u\left(x+f_{1}(p) p\right) u\left(x-\left(1-f_{1}(p)\right) p\right) d p d x \\
& +\int_{\mathbb{R}} \int_{\mathbb{R}} \pi_{2}(p) \phi(x) u\left(x+f_{2}(p) p\right) u\left(x-\left(1-f_{2}(p)\right) p\right) d p d x \\
& -\int_{\mathbb{R}} \int_{\mathbb{R}} \phi(x) u(x) u(p) d x d p
\end{aligned}
$$

By using Fubini's theorem we have

$$
\begin{aligned}
V= & \int_{\mathbb{R}} \int_{\mathbb{R}} \pi_{1}(p) \phi(x) u\left(x+f_{1}(p) p\right) u\left(x-\left(1-f_{1}(p)\right) p\right) d x d p \\
& +\int_{\mathbb{R}} \int_{\mathbb{R}} \pi_{2}(p) \phi(x) u\left(x+f_{2}(p) p\right) u\left(x-\left(1-f_{2}(p)\right) p\right) d x d p \\
& -\int_{\mathbb{R}} \int_{\mathbb{R}} \frac{\phi(x)+\phi(p)}{2} u(p) u(x) d x d p
\end{aligned}
$$

By using the changes of variable, choosing the variable $\mathrm{y}$ instead of $\mathrm{x}$ with $y=x+f_{1}(p) p, y=x+\left(1-f_{2}\right)(p) p$ and $y=x-p$ respectively in the first, the second and the third integral we obtain

$$
\begin{aligned}
V & =\int_{\mathbb{R}} \int_{\mathbb{R}}\left[\pi_{1}(l) \phi\left(y+f_{1}(p) p\right)+\pi_{2}(p) \phi\left(y+l-f_{2}(p) p\right)\right. \\
& \left.-\frac{\phi(y)+\phi(y+p)}{2}\right] u(y) u(y+p) d p d y
\end{aligned}
$$

Therefore $V=0$ whenever

$$
\pi_{1}(l) \phi\left(y+f_{1}(p) p\right)+\pi_{2}(p) \phi\left(y+l-f_{2}(p) p\right) \equiv \frac{\phi(y)+\phi(y+p)}{2} .
$$

To conclude it is sufficient to verify the above equality respectively when $\phi(x) \equiv$ 1 and $\phi(x)=x$ and we obtain $(i)$ and $(i i)$.

By using the same arguments as in Hinow et al. 11] combined with Lemma 1355 we have the following result. 
Theorem 7 Let $\tau>0$. Let Assumptions 2 and 3 be satisfied. For each initial distribution $u_{0} \in L_{+}^{1}(\mathbb{R})$ (3.4) has a unique global positive solution $u(t,$.$) .$ Moreover the first and second moments of the distribution $u_{0}$ are preserved in time. Namely we have the following properties for each $t \geq 0$

$$
\int_{\mathbb{R}} u(t, x) d x=\int_{\mathbb{R}} u_{0}(x) d x \text { and } \int_{\mathbb{R}} x u(t, x) d x=\int_{\mathbb{R}} x u_{0}(x) d x .
$$

Remark 8 The differential equation (3.4) also preserves the support of the distribution $u_{0} \in L_{+}^{1}(\mathbb{R})$. That is to say that if support $\left(u_{0}\right) \subset I$ (for an interval $I \subset \mathbb{R})$ then for each $t \geq 0$

$$
\operatorname{support}(u(t, .)) \subset I \text {. }
$$

Remark 9 By using the same modelling ideas than in Ducrot et al. [7], we could include the cell proliferation as follows

$$
\partial_{t} u(t, p)=2 \tau[T(u(t, .))(x)-u(t, x)]+\left[\frac{\beta}{1+\gamma U(t)}-\mu\right] u(t, p), \text { for } x \in \mathbb{R},
$$

where $U(t)=\int_{\mathbb{R}} u(t, p) d p$ is the total number of cells, with $\beta>0$ is the division and $\mu>0$ is the mortality rate. The fraction $\frac{1}{1+\gamma U}$ is taking into account the contact inhibition pressure. The above model is not taking into account the time between two cell divisions. It is still a modeling issue to take into account the cell cycle into such a transfer model. In particular, we would be to know how $P-g p$ is redistributed between the daughter cells (at cell division).

\section{The Discrete Model}

Given the maximal value of the transferable quantity $x_{\max }$ and its minimal value $x_{\text {min }}$, we consider a partition

$$
0=x_{1}<x_{2}<\ldots<x_{i}=x_{1}+i \Delta x<\ldots<x_{I_{L}}=L
$$

with $x_{i+1}-x_{i}=\Delta x, \forall i=1, I_{L}-1$. Starting with the initial distribution $u_{0}(x)$ for all sizes $x$, we introduce an initial sequence $u_{i}^{0}, i=1, \ldots I_{L}$, by

$$
u_{i}^{0}=\frac{1}{\Delta x} \int_{x_{i}}^{x_{i+1}} u_{0}(x) d x \simeq u_{0}\left(\frac{x_{i+1}+x_{i}}{2}\right) .
$$

We suppose that all the values $u_{i}^{n}$ for $i=1, \ldots, I_{L}$ are known, and we propose to build the $u_{i}^{n+1}$ for $i=1, \ldots I_{L}$ by the following scheme:

$$
\begin{aligned}
u_{i}^{n+1}=u_{i}^{n} & +2 \tau \Delta t \frac{1}{\left(\sum_{j=1}^{I_{L}} u_{j}^{n} \Delta x\right)} \sum_{j \in \mathbb{Z}} \pi_{1}\left(l_{j}\right) \bar{u}_{i-f_{1}\left(p_{j}\right) j}^{n} \bar{u}_{i+\left(1-f_{1}\left(p_{j}\right)\right) j}^{n} \Delta x \\
& +2 \tau \Delta t \frac{1}{\left(\sum_{j=1}^{I_{L}} u_{j}^{n} \Delta x\right)} \sum_{j \in \mathbb{Z}} \pi_{2}\left(p_{j}\right) \bar{u}_{i-\left(1-f_{2}\left(p_{j}\right)\right) j}^{n} \bar{u}_{i+f_{2}\left(p_{j}\right) j}^{n} \Delta x \\
& -2 \tau \Delta t u_{i}^{n}
\end{aligned}
$$


where $p_{j}=j \Delta x$ is the difference of the transferable quantities between two partner cells, the quantities $\left.i-f_{1}\left(p_{j}\right)\right) j, i+\left(1-f_{1}\left(p_{j}\right) j, i-f_{2}\left(p_{j}\right) j\right.$ and $i+(1-$ $f_{2}\left(p_{j}\right) j$ are understood respectively as their integer parts in the system 4.1), and

$$
\bar{u}_{i}:=\left\{\begin{array}{l}
u_{i} \text { if } i=1, \ldots, I_{L}, \\
0 \text { otherwise. }
\end{array}\right.
$$

Let us rewrite the above scheme (4.1) under the compact form

$$
u_{i}^{n+1}=u_{i}^{n}+2 \tau \Delta t F_{i}^{n}
$$

with

$$
F_{i}^{n}:=\frac{\sum_{j} \sum_{k} K_{i j k} u_{j}^{n} u_{k}^{n}}{\sum_{j=1} u_{j}^{n}}-u_{i}^{n}
$$

where $K_{i j k}$ will be defined precisely in section 5 .

Similarly to the continuous case, for any sequence $\left(\phi_{i}\right)_{i=1, \ldots, I_{L}}$ we have the following inequality

$$
\sum_{j=1}^{I_{L}} u_{j}^{n} \sum_{i=1}^{I_{L}} \phi_{i} F_{i}^{n}=\sum_{j=1}^{I_{L}} \sum_{k=1}^{I_{L}}\left(\sum_{i=1}^{I_{L}} K_{i, j, k} \phi_{i}-\frac{\phi_{j}+\phi_{k}}{2}\right) u_{j}^{n} u_{k}^{n} .
$$

Therefore

$$
\sum_{i=1}^{I_{L}} \phi_{i} F_{i}^{n}=0, \forall u^{0} \geq 0
$$

if and only if

$$
\sum_{i=1}^{I_{L}} \phi_{i} K_{i, j, k}=\frac{\phi_{j}+\phi_{k}}{2}
$$

Theorem 10 The total number of cells $\sum_{i=1}^{I_{L}} u_{i}^{n}$ is constant in time for the solution of the discrete model (4.2) if and only if

$$
\sum_{i=1}^{I_{L}} K_{i, j, k}=1, \forall j, k=1, \ldots, I_{L}
$$

The total mass of transferable quantities is preserved in time for the solution of the discrete model (4.2) if and only if

$$
\sum_{i=1}^{I_{L}} x_{i} K_{i, j, k}=\frac{x_{j}+x_{k}}{2}
$$




\section{Simulation experiments}

In this section, we present some numerical simulations to calibrate the transfer model (3.4) with experimental data used in [14]. The numerical scheme presented in the above section was implemented using MATLAB.

\subsection{Material and method}

\subsubsection{Transfer kernel}

According to Assumption 3 if a cell $C_{j}$ and a cell $C_{k}$ have, respectively, a quantity $x_{j}$ and $x_{k}$ of P-gp activity before transfer with $x_{j} \geq x_{k}$, then, after transfer, $C_{j}$ (respectively, $C_{k}$ ) will have an activity $x_{j}-\left(x_{j}-x_{k}\right) f_{1}\left(x_{j}-x_{k}\right)$ with the probability $\pi_{1}\left(x_{j}-x_{k}\right)$ (respectively, $x_{k}+\left(x_{j}-x_{k}\right) f_{2}\left(x_{j}-x_{k}\right)$ with 160 the probability $\pi_{2}\left(x_{j}-x_{k}\right)$ ). So the fraction transferred is $f_{1}\left(x_{j}-x_{k}\right)$, with a probability $\pi_{1}\left(x_{j}-x_{k}\right)$ (resp. $\pi_{2}\left(x_{j}-x_{k}\right)$ ) for $C_{j}$ (respectively, $C_{k}$ ) which depends on the difference between $x_{j}$ and $x_{k}$ (the distance between the P-gp activities of $C_{j}$ and $C_{k}$ ).

Together with these considerations, one can define the transfer kernel $K$ satisfying the properties of Theorem 10 as follows:

$$
K_{i, j, k}:=\left\{\begin{array}{l}
\pi_{1}\left(x_{j}-x_{k}\right) \text { if } x_{i}=x_{j}-\left(x_{j}-x_{k}\right) f_{1}\left(x_{j}-x_{k}\right), \\
\pi_{2}\left(x_{j}-x_{k}\right) \text { if } x_{i}=x_{k}+\left(x_{j}-x_{k}\right) f_{2}\left(x_{j}-x_{k}\right), \\
0 \text { otherwise. }
\end{array}\right.
$$

In what follows, we simplify the transfer efficiency $f_{1}$ and $f_{2}$ by assuming that

$$
f_{1}\left(x_{j}-x_{k}\right)=\left\{\begin{array}{lc}
\bar{f}_{1} & \text { if }\left(x_{j}-x_{k}\right) \in\left(\delta_{\min }, \delta_{\max }\right) \\
\epsilon \bar{f}_{1} & \text { otherwise }
\end{array}\right.
$$

and

$$
f_{2}\left(x_{j}-x_{k}\right)= \begin{cases}\bar{f}_{2} & \text { if }\left(x_{j}-x_{k}\right) \in\left(\delta_{\min }, \delta_{\max }\right) \\ \epsilon \bar{f}_{2} & \text { otherwise. }\end{cases}
$$

Remark $11 \epsilon=0$ means that the transfer occurs only if $\delta_{\min }<x_{j}-x_{k}<\delta_{\max }$. $\epsilon \in(0,1)$ means that a low quantity is transferred when the difference $x_{j}-x_{k}$ is either very low or very large.

\subsubsection{Data scaling: data obtained by Flow cytometry}

170 To calibrate the transfer model in the section below, we use the data described in [14] where experimentally 50\% of non P-gp-expressing parental MCF-7 cells were co-cultured with $50 \%$ of their multidrug resistant counterpart MCF-7/Doxo variants of the human breast cancer cell line cf. [14]. Flow cytometry analysis 
of efflux activity transfers between MCF-7 and MCF-7/Doxo was considered. Membrane P-gp content was followed over time, from day 0 to day 6 , by flow cytometry. After $0,3,4,5$ and 6 days of co-culture a sample of 10000 cells was analyzed. Experimental data obtained were expressed in fluorescence units (f.u). According to the fluorescence $(\mathrm{F})$ scale used in cytometry, $F$ varies between $F_{\min }=1$ and $F_{\max }=10^{4}$.

We point out that fluorescence scale was not suitable to calibrate the transfer model for two reasons:

(i) the distance between the P-gp activities of two cells $C_{j}$ and $C_{k}$ having respectively a quantity $x_{j}$ and $x_{k}$ of $\mathrm{P}$-gp activity may be inappropriate because the distance considered, is defined by $d\left(C_{j}, C_{k}\right)=\left|x_{j}-x_{k}\right|$. The result could be underestimate.

(ii) The model was written in terms of transferable quantity, (e.g. transfers of P-glycoproteins) while experimental data give cell fluorescence accumulation as an inverse function of P-gp activity cf. [14.

In order to deal with (i)-(ii), fluorescence units were rescaling into an arbitrary P-glycoproteins unit by introducing a non-linear bijection map $F:[0,1] \rightarrow$ $\left[1,10^{4}\right]$ defined by $F(p)=10^{4 p}$ where $p$ denotes the P-glycoproteins expression on the cell surface and $F$ its corresponding fluorescence value.

\subsubsection{Estimation of model parameters}

We selected for model validation two scenarios: in both cases, the standard error $195(R M S E)$ of simulated and observed data was estimated. Recall that $R M S E:=$ $\sqrt{\sum_{l=1}^{N}\left(o b s\left(t_{l}\right)-\operatorname{sim}\left(t_{l}\right)\right)^{2} / N}$ where $N$ is the number of observed data, $o b s\left(t_{l}\right)$ and $\operatorname{sim}\left(t_{l}\right)$ are the observed and simulated data at time $t_{l}$ respectively.

Scenario 1 (unequal fractions transferred): we assume that transfer fractions $\bar{f}_{1}$ and $\bar{f}_{2}$ defined in $(5.2)-(5.3)$ are different and the fitting parameters are carried out by the minimization of the RMSE.

Scenario 2 (equal fractions transferred): we assume that transfer fractions $\bar{f}_{1}$ and $\bar{f}_{2}$ defined in (5.2)-(5.3) are equal and the fitting parameters are carried out by the minimization of the RMSE. 


\begin{tabular}{llllll}
\hline Symbol & Description & Scen. 1 & Scen. 2 & Units & Method \\
\hline \hline$\tau$ & Rate of transfer of P-gp activity & 0.95 & 0.90 & day $^{-1}$ & fitted \\
$F_{\min }$ & Minimum value of P-gp activity & 1 & 1 & f.u & data \\
$F_{\max }$ & Maximum value of P-gp activity & $10^{4}$ & $10^{4}$ & f.u & data \\
$\delta_{\min }$ & Minimum threshold for transfers & $10^{4 * 0.41}$ & $10^{4 * 0.32}$ & f.u & fitted \\
$\delta_{\max }$ & Maximum threshold for transfers & $10^{4 * 0.46}$ & $10^{4 * 0.63}$ & f.u & fitted \\
$\bar{f}_{1}$ & Transfer efficiency for p value in $J$ & 0.48 & 0.1 & - & fitted \\
$\bar{f}_{2}$ & Transfer efficiency for p value in $J$ & 0.07 & 0.1 & - & fitted \\
$\epsilon \bar{f}_{1}$ & Transfer efficiency for p value in $J^{c}$ & 0.05 & 0.001 & - & fitted \\
$\epsilon \bar{f}_{2}$ & Transfer efficiency for p value in $J^{c}$ & 0.007 & 0.001 & - & fitted \\
\hline \hline
\end{tabular}

Table 1: List of the model parameters, their significations, values and symbols. The model is simulated with an arbitrary P-glycoproteins unit $p \in[0,1]$ and by using a rescaling argument $F(p):=10^{4 p} \in\left[1,10^{4}\right]$ one gets its equivalent in terms of fluorescence units (f.u). Here $J:=\left(\delta_{\min }, \delta_{\max }\right)$ and $J^{c}$ its complementary.

\subsection{Results}

\subsubsection{Data scaling: data obtained by Flow cytometry}

Results after experimental data scaling are shown in Figure 3 
(a)

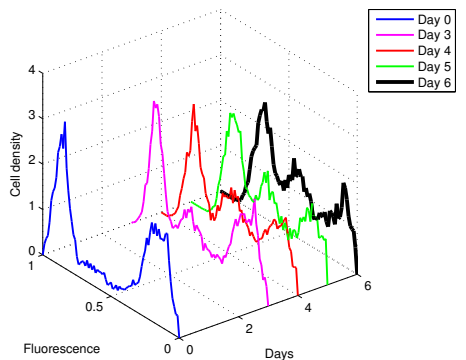

(b)

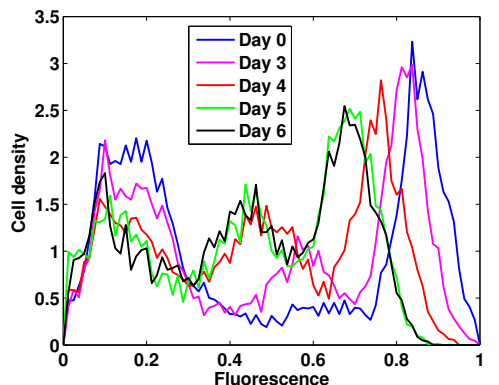

Figure 3: Flow cytometry analysis of efflux activity transfers between MCF-7 and MCF-7/Doxo variants of the human breast cancer cell line. 50:50 MCF 7:MCF 7/Doxo cell mixture is seeded on culture dishes at day 0 and co-cultured during 6 days. After 0, 3, 4, 5 and 6 days of co-culture a sample of 10000 cells was analysed. Figures (a) and (b) are two different types of visualisation of the same distribution in time. Figure (a) looks clearer, but the figure (b) will be more convenient to data with the models. One may observe that the larger the fluorescence is the less the cells are capable to efflux calceinAM. Therefore the sensitive cells sensitive to drug treatment are located on the right side of figure (b), while the cells multi-drug resistant are located on the left side of figure (b).

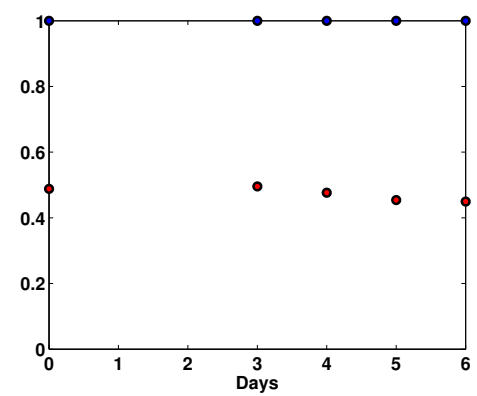

Figure 4: The blue dots represent the integral over $\left[x_{\min }, x_{\max }\right]$ of the P-gp distribution which is 1 since the distribution coming from the measurement has been renormalized (by dividing by 10000 the total number of cells measured). The red dots represent the total mass of fluorescence at day 0, 3, 4, 5 and 6. The total mass of fluorescence stays around 0.5 during the experiment.

\subsubsection{Estimation of model parameters}

Scenario 1 (Unequal fraction transferred): after the minimization of the $R M S E$ considering unequal fraction transferred, we get $\bar{f}_{1}=0.48$ and $\bar{f}_{2}=0.07$, the rate 

maximum threshold for large transfer is $\delta_{\max }=0.46$, the low transferred quantity when the difference between the donor and the recipient is either very low or very large is $\epsilon \bar{f}_{1}=0.0504$ and $\epsilon \bar{f}_{2}=0.0074$. Simulated results are shown in figure 5 (a), $R M S E=0.2854$.

Scenario 2 (Equal fraction transferred): after the minimization of the $R M S E$ considering Equal fraction transferred, we get $\bar{f}_{1}=\bar{f}_{2}=0.1$, the rate of transfer, $\tau=0.90$, the minimum threshold for large transfer, $\delta_{\min }=0.32$ and the maximum threshold for large transfer, $\delta_{\max }=0.63, \epsilon \bar{f}_{1}=0.001$ and $\epsilon \bar{f}_{2}=0.001$. Simulated results are shown in Figure 5 (b), $R S M E=0.5518$.

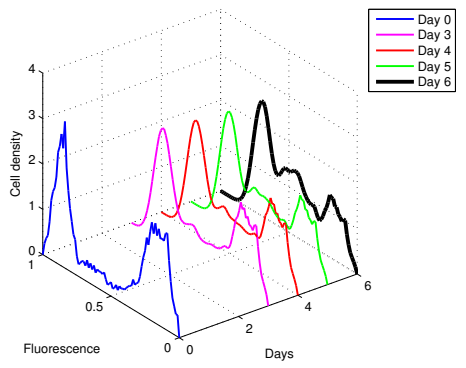

(a) $R S M E=0.2854$

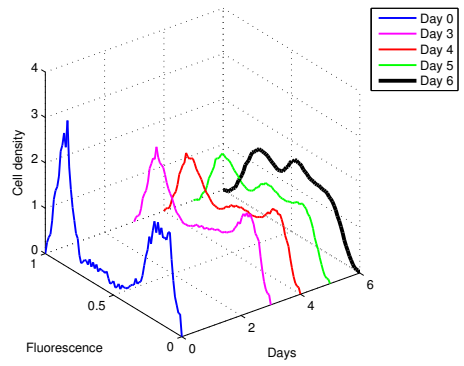

(a) $R S M E=0.5518$

Figure 5: Evolution of cell density with respect to the quantity of P-glycoproteins. Experimental results showing the distribution of the cell density with respect to the quantity of P-glycoproteins are shown in Figure 3. (a) Simulated results when transfer fractions $\bar{f}_{1}$ and $\bar{f}_{2}$ are different: $\bar{f}_{1}=0.48$ and $\bar{f}_{2}=0.07$. (b) Simulated results when transfer fractions $\bar{f}_{1}$ and $\bar{f}_{2}$ are assumed equal: $\bar{f}_{1}=$ $\overline{f_{2}}=0.1$,

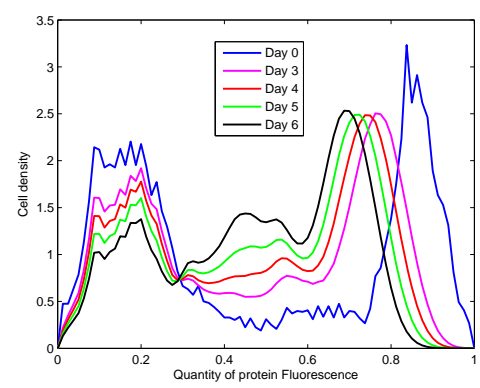

(a) $R S M E=0.2854$

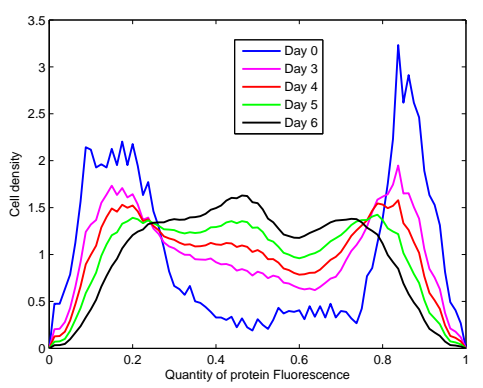

(b) $R S M E=0.5518$

Figure 6: Evolution of cell density with respect to the quantity of P-glycoproteins. Experimental results showing the distribution of the cell density with respect to the quantity of P-glycoproteins are shown in Figure 3. This figure is another different type of visualisation of the Figure 5 
By comparing the RMSE values for both scenarios, one can conclude that the first scenario gives a good fit of data compared to the second one. Since experimental data used to fit our model were already used to fit a model developped in 11, 14, our simulation results and those obtained in 14 are very close when we take the same transfer rule, namely $\pi_{1}=\pi_{2}=1 / 2$, but it does not provide a good fit of experimental data. So our model extends the previous model in [11, 14] where only equal fraction transferred was possible.

\section{Directions for future experimental studies}

The model presented here, in the absence of precise values for the multitude of physprovide some qualitative predictions. Careful experimental studies may validate or correct certain aspects of this model. These predictions and assumptions should help to tease out the role the direct transfer of proteins plays physiologically. We have a simplistic constant rate of transfer. Imaging a large number of cells could help to 235 provide us with a better understanding of the dynamic of direct transfer formation and their static distribution. Experiments measuring the protein transfer of differing sizes can shed light on the validity of our model.

\section{Conclusion}

In this paper, a model was formulated to describe some transfer rules in popula240 tion dynamics. Population was structured by a continuous variable corresponding to transferable material while the time was considered continuous. The model extends the previous model developed in [11, 14].

Mathematical analysis was performed, namely existence and uniqueness of solutions, the preservation of individuals number, the preservation of transfer quantity mass. Moreover a discrete version of the model was also presented.

The model was able to reproduce experimental data obtained from experiments on the consequences of cell-to-cell protein transfer in breast cancer.

Our model can be extended to include spatial organization of population as well as population growth (without limit cycle description see [7]). Our model provides an 250 alternative to carrying out further large-scale studies concerning transfer processes in population dynamics. Our results are applicable generally to systems in which transfer of quantities occurs within a population. 


\section{Appendix: Convergence of the discrete model to continuous model}

In this section we show the convergence of the scheme 4.1 . It means that the difference $\left|u-u^{\Delta}\right|_{1}$ between the approximate solution $u^{\Delta}$ of the discrete model (4.1) and the solution $u$ of the continuous model (3.4) tends to zero as the meshsizes $\Delta t, \Delta x$ go to zero (for simplicity we set $\Delta t=r \Delta x$ ). Here the approximate solution $u^{\Delta}$ is defined as the piecewise constant function defined on $] 0, T\left[\times \Omega_{L}\right.$ (with $\Omega_{L}:=[0, L]$ )

$$
\left.u^{\Delta}(t, x)=u_{i}^{n} \text { For all }(t, x) \in,\right] t_{n}, t_{n+1}[\times] x_{i}, x_{i+1}[
$$

where the values $u_{i}^{n}$ are computed by 4.1).

Theorem 12 Let $u_{0} \in\left(L^{1} \cap L^{\infty}\right)\left(\Omega_{L}\right)$ with total variation be bounded locally in $\Omega_{L}$, $u_{0} \geq 0$, then as the meshsize $\Delta x$ tends to zero, there is a subsequence of $\left(u^{\Delta}\right)_{\Delta x>0}$, the family of approximate solution, converging in $L_{l o c}^{1}\left([0, T] \times \Omega_{L}\right)$ to a function $u \in$ $L_{l o c}^{1}\left([0, T] \times \Omega_{L}\right)$.

260 The limiting function $u(t, x)$ just obtained is a weak solution of the problem (3.1).

On order to prove Theorem 12 we first prove the existence of a limit $u$ to $u^{\Delta}$ when the meshsize $\Delta x$ goes to zero. Then we prove that this limit is a solution of the continuous problem 3.1.

Existence of a limit for $u^{\Delta}$ : The proof of existence of the limit $u(t, x)$ is based on the compact canonical imbedding from $W^{1,1}(\Omega)$ into $L^{1}(\Omega)$. Let $I\left(u^{\Delta}\right)$, defined on $[0, T] \times \Omega_{L}$, be the interpolate of degree one of $u^{\Delta}$ at the vertices of each rectangle $\left[x_{i}, x_{i+1}\right] \times\left[t_{n}, t_{n+1}\right]$ where it is given by

$$
\begin{aligned}
I\left(u^{\Delta}\right)(x, t)= & u_{i}^{n}+\left(u_{i+1}^{n}-u_{i}^{n}\right) \frac{x-i \Delta x}{\Delta x}+\left(u_{i}^{n+1}-u_{i}^{n}\right) \frac{t-n r \Delta x}{r \Delta x} \\
& +\left(u_{i+1}^{n+1}-u_{i}^{n+1}-u_{i+1}^{n}+u_{i}^{n}\right) \frac{(x-i \Delta x)(t-n r \Delta x)}{r(\Delta x)^{2}}
\end{aligned}
$$

$I\left(u^{\Delta}\right)$ is continuous with

$$
\left|I\left(u^{\Delta}\right)\right|_{L^{\infty}\left([0, T] \times \Omega_{L}\right)}=\left|u^{\Delta}\right|_{L^{\infty}\left([0, T] \times \Omega_{L}\right)}=\sup _{n, i}\left|u_{i}^{n}\right|
$$

and differentiable inside each rectangle. Thus we obtain

$$
\iint\left|\frac{\partial I\left(u^{\Delta}\right)}{\partial t}\right| d x d t \leq \sum_{n=0}^{N} \sum_{i \leq I_{L}}\left|u_{i}^{n+1}-u_{i}^{n}\right| \Delta x .
$$

In the same way

$$
\iint\left|\frac{\partial I\left(u^{\Delta}\right)}{\partial x}\right| d x d t=\sum_{n=0}^{N} \sum_{i \leq I_{L}}\left|u_{i+1}^{n}-u_{i}^{n}\right| r \Delta x .
$$

On the other hand one can check that the numerical scheme 4.1 satisfies the following a priori estimates 


$$
\begin{aligned}
& \sup _{i}\left|u_{i}^{n+1}\right| \leq\left(1+C_{1} \Delta t\right) \sup _{i}\left|u_{i}^{n}\right| \\
& \sum_{i=1}^{I_{L}-1}\left|u_{i+1}^{n+1}-u_{i}^{n+1}\right| \leq\left(1+C_{2} \Delta t\right) \sum_{i=1}^{I_{L}-1}\left|u_{i+1}^{n}-u_{i}^{n}\right| \\
& \sum_{i=1}^{I_{L}-1}\left|u_{i}^{n+1}-u_{i}^{n}\right| \leq \sum_{i=1}^{I_{L}-1}\left|u_{i+1}^{n}-u_{i}^{n}\right|
\end{aligned}
$$

where $C_{1}$ and $C_{2}$ are two constants independent of $\mathrm{n}$.

Let $u_{0} \in L^{\infty}\left(\Omega_{L}\right)$, then $\sup _{i}\left|u_{i}^{0}\right| \leq C$. It follows that $u^{\Delta}$ is bounded and then 270 contains a subsequence $u^{\Delta_{p}}$ weakly star convergent to a limit $u \in L^{\infty}\left([0, T] \times \Omega_{L}\right)$ bounded by $\left|u_{0}\right|_{L}^{\infty}\left(\Omega_{L}\right)$.

Let $u_{0}$ with the total variation $T V\left(u_{0}(x)\right)=\sum_{i=1}^{I_{L}}\left|u_{0}\left(x_{i+1}\right)-u_{0}\left(x_{i}\right)\right|$ be bounded, we have,by applying the discrete Gronwall lemma to the estimates 8.5 and using successively 8.2 and $8.3,8.4$, It follows also that

$$
\left|I\left(u^{\Delta}\right)\right|_{L^{\infty}\left([0, T] \times \Omega_{L}\right)}+\left|\frac{\partial I\left(u^{\Delta}\right)}{\partial x}\right|_{L^{1}\left([0, T] \times \Omega_{L}\right)}+\left|\frac{\partial I\left(u^{\Delta}\right)}{\partial t}\right|_{L^{1}\left([0, T] \times \Omega_{L}\right)} \leq M .
$$

Therefore from $\left\{I(u)^{\Delta}\right\}$ associated to $\left\{u^{\Delta}\right\}$, we extract a subsequence convergent to $\left\{I(u)^{\Delta_{p}}\right\}$ in $L_{l o c}^{1}(] 0, T\left[\times \Omega_{L}\right)$. Then we verify that $\left\{I(u)^{\Delta_{p}}-u^{\Delta_{p}}\right\}$ tends to zero in $L^{1}$ , for all bounded open sets $] 0, T\left[\times \Omega_{L}\right.$. Since the associate subsequence $u^{\Delta}$ weakly star converges to a function $u \in L^{\infty}(] 0, T\left[\times \Omega_{L}\right)$, and since on the other hand $\left\{I\left(u^{\Delta_{p}}\right)\right\}$ is convergent in $L_{l o c}^{1}(] 0, T\left[\times \Omega_{L}\right)$, we have

$$
u^{\Delta} \text { converges to } u \text { in } L_{l o c}^{1}(] 0, T\left[\times \Omega_{L}\right) .
$$

This ends the proof of the existence of a limit.

Convergence of $F^{\Delta}\left(u^{\Delta}\right)$ to $T(u)$ : Let us define the discrete operator acting on the piecewise constant function $u^{\Delta}$ by

$$
\left.F^{\Delta}\left(u^{\Delta}\right)=F_{i}^{n} \text { for all }(t, x) \in,\right] t_{n}, t_{n+1}[\times] x_{i}, x_{i+1}[
$$

where $F_{i}^{n}$ given by 4.3 . We have proved the following lemma

Lemma 13 The discrete transfer operator $F^{\Delta}\left(u^{\Delta}\right)$ converges to the continuous trans275

fer operator $T(u), u$ being the limit function of $u^{\Delta}$ as $\Delta x$ goes to zero.

Let us write

$$
\left|F^{, \Delta}\left(u^{\Delta}\right)-T(u)\right|_{1} \leq\left|F^{\Delta}\left(u^{\Delta}\right)-T\left(u^{\Delta}\right)\right|_{1}+\left|T\left(u^{\Delta}\right)-T(u)\right|_{1}
$$

Since The transfer operator $\mathrm{T}$ is Lipchitz and $u^{\Delta}$ converges to $u$ the second term of the right hand side of the above inequality $\int_{0}^{1}\left|T^{\Delta}\left(u^{\Delta}\right)(x)-T(u)(x)\right| d x \longrightarrow 0$ as the meshsize $\Delta x$ goes to zero. 
Let us now calculate $\widehat{T}\left(u^{\Delta}\right)(x)$.

$$
\begin{aligned}
\widehat{T}\left(u^{\Delta}\right)(x) & =\sum_{j} \int_{x_{j}}^{x_{j+1}} \pi_{1}(p) u^{\Delta}\left(x_{i}+f_{1}(p) p\right) u^{\Delta}\left(x_{i}-\left(1-f_{1}(p)\right) p\right) d p \\
& +\sum_{j} \int_{x_{j}}^{x_{j+1}} \pi_{2}(p) u^{\Delta}\left(x_{i}+f_{2}(p) p\right) u^{\Delta}\left(x_{i}-\left(1-f_{2}(p)\right) p\right) d p . \\
& =\sum_{j} \int_{x_{j}}^{x_{j+1}} \pi_{1}(p) d p u_{i+f_{1}\left(p_{j}\right) j}^{n} u_{i-\left(1-f_{1}\left(p_{j}\right)\right) j}^{n} \\
& +\sum_{j} \int_{x_{j}}^{x_{j+1}} \pi_{2}(p) d p \bar{u}_{i+f_{2}\left(p_{j}\right) j}^{n} \bar{u}_{i-\left(1-f_{2}\left(p_{j}\right)\right) j .}^{n} .
\end{aligned}
$$

In section 3 we use the approximation $\pi_{1}$ and $\pi_{2}$

$$
\pi_{1}\left(l_{j}\right)=\frac{\int_{x_{j}}^{x_{j+1}} \pi_{1}(p) d p}{\Delta x} \text { and } \pi_{2}\left(l_{j}\right)=\frac{\int_{x_{j}}^{x_{j+1}} \pi_{2}(p) d p}{\Delta x}
$$

we conclude that $T\left(u^{\Delta}\right)-F^{\Delta}\left(u^{\Delta}\right)=0$. This completes the proof of the lemma.

Weak solution: Now we consider the consistancy of the scheme, which means that this limit $\mathrm{u}$ is a weak solution of the continuous problem 3.1 . For all smooth $\phi \in$ $C^{1}\left([0, T] \times \Omega_{L}\right)$ with compact support in $[0, T[\times[0,1]$, we define

$$
\forall(t, x) \in\left[x_{i-1}, x_{i}\left[\times\left[t_{n}, t_{n+1}\left[, \quad \phi^{\Delta}(t, x)=\phi_{i}^{n}=\frac{1}{\Delta t \Delta x} \int_{t_{n}}^{t_{n+1}} \int_{x_{i-1}}^{x_{i}} \phi(t, x) d t d x .\right.\right.\right.\right.
$$

Multiplying the scheme 4.1 by $\Delta x \phi_{i}^{n}$ we get,

$$
\sum_{i, n}\left(u_{i}^{n+1}-u_{i}^{n}\right) \phi_{i}^{n}-2 \tau \Delta t \sum_{i, n} F_{i}^{n} \phi_{i}^{n} \Delta x=0,
$$

then summing by part we get

$$
\sum_{i, n}\left(u_{i}^{n+1}\left(\phi_{i}^{n}-\phi_{i}^{n+1}\right)\right) \Delta x-2 \tau \Delta t \sum_{i, n} F_{i}^{n} \phi_{i}^{n} \Delta x-\sum_{i} u_{i}^{0} \phi_{i}^{0} \Delta x=0
$$

which is equivalent to

$$
\begin{gathered}
\int_{0}^{T} \int_{\Omega_{L}} u^{\Delta}(t, x) \frac{\phi^{\Delta}(t+\Delta t, x)-\phi^{\Delta}(t, x)}{\Delta t} d x d t+\int_{0}^{T} \int_{\Omega_{L}} 2 \tau F^{\Delta}\left(u^{\Delta}\right)(t, x) \phi^{\Delta}(t, x) d x d t \\
+\int_{\Omega_{L}} u^{\Delta}(0, x) \phi^{\Delta}(0, x) d x=0
\end{gathered}
$$

we pass to the limit $\Delta x \rightarrow 0$, we obtain

$$
\begin{aligned}
& \int_{0}^{T} \int_{\Omega_{L}} u(t, x) \frac{\partial \phi}{\partial t}(t, x) d x d t+\int_{0}^{T} \int_{\Omega_{L}} 2 \tau[T(u(t, .))(x)-u(t, x)] \phi(t, x) d x d t \\
& +\int_{\Omega_{L}} u(0, x) \phi(0, x) d x=0
\end{aligned}
$$

which means that the limit $\mathrm{u}$ obtained using the discrete scheme is a weak solution of the problem 3.1 with the initial data $u_{0}(x)$. 


\section{References}

[1] S. V. Ambudkar, Z. E. Sauna, M. M. Gottesman, and G. Szakacs, A novel way to spread drug resistance in tumor cells: Functional intercellular transfer of Pglycoprotein (ABCB1), Trends Pharmacol. Sci., 26 (2005), 385-387.

[2] N. Bellomo and M. Delitala, From the mathematical kinetic, and stochastic game theory to modelling mutations, onset, progression and immune competition of cancer cells, Physics of Life Reviews, 5 (2008), 183-206.

[3] N. Bellomo, N. Li, and P. Maini, On the foundations of cancer modelling selected topics, speculations, and perspectives, Mathematical Models and Methods in Applied Sciences, 18 (2008), 593-646.

[4] A. Bellouquid and M. Delitala, Mathematical Modeling of Complex Biological Systems: A Kinetic Theory Approach, Birkhäuser, Boston, Basel, Berlin, 2006.

[5] B. Dale, G. P. McNerney, D. L. Thompson, W. Hübner, T. Huser, B. K. Chen, Visualizing Cell-to-cell Transfer of HIV using Fluorescent Clones of HIV and Live Confocal Microscopy, JoVE (2010) 44.

[6] D.M. Davis et al. The protean immune cell synapse: a supramolecular structure with many functions. Semin. Immunol. 15 (2003), 317-324.

[7] A. Ducrot, F. Le Foll, P. Magal, H. Murakawa, J. Pasquier, G. F. Webb, An in vitro cell population dynamics model incorporating cell size, quiescence, and contact inhibition, Mathematical Models and Methods in Applied Sciences 21 (2011), 871-892.

[8] M. E. Dudley and S. A. Rosenberg, Adoptive-cell-transfer therapy for the treatment of patients with cancer, Nature Rev. Cancer 3 (2002), 666-675.

[9] M. E. Dudley, et al. Adoptive cell transfer therapy following non-myeloablative but lymphodepleting chemotherapy for the treatment of patients with refractory metastatic melanoma, J. Clin. Oncol. 23 (2005), pp. 2346-2357.

[10] C. Hansen, E. Angot, A. L. Bergstrom, J.A. Steiner, L. Pieri, G. Paul, T.F. Outeiro, R. Melki, P. Kallunki, K. Fog, J. Y. Li, P. Brundin, $\alpha$-Synuclein propagates from mouse brain to grafted dopaminergic neurons and seeds aggregation in cultured human cells, J Clin Invest 121 (2011), 715-725.

315 [11] P. Hinow, F. Le Foll, P. Magal, G. F. Webb, Analysis of a model for transfer phenomena in biological populations, SIAM J. Appl. Math. 70 (2009), 40-62.

[12] A. Levchenko, B. M. Mehta, X. Niu, G. Kang, L. Villafania, D. Way, D. Polycarpe, M. Sadelain, and S. M. Larson, Intercellular transfer of P-glycoprotein mediates acquired multidrug resistance in tumor cells, Proc. Natl. Acad. Sci. USA, 102 (2005), 1933-1938.

[13] A. S. Novozhilov, G. P. Karev, and E. Koonin, Mathematical modeling of evolution of horizontally transferred genes, Mol. Biol. Evol., 22 (2005), 1721-1732.

[14] J. Pasquier, P. Magal, C. Boulangé-Lecomte, G. Webb and F. Le Foll, Consequences of cell-to-cell P-glycoprotein transfer on acquired multidrug resistance in breast cancer: a cell population dynamics model, Biol. Direct. 6:5 (2011).

[15] J. Pasquier, L. Galas, C. Boulang-Lecomte, D. Rioult, F. Bultelle, P. Magal, G. Webb and F. Le Foll (2012), Different modalities of intercellular membrane exchanges mediate cell-to-cell P-glycoprotein transfers in MCF-7 breast cancer cells, Journal of Biological Chemistry 287(10) (2012), 7374-7387. 
[16] Y. Suhail, J. Lee, M. Walker, D. H. Kim, M. D. Brennan, J. S. Bader, and A. Levchenko, Modeling intercellular transfer of biomolecules through tunneling nanotubes. Bulletin of mathematical biology, 75(8) (2013), 1400-1416.

[17] C. Villani, A review of mathematical topics in collisional kinetic theory, in Handbook of Mathematical Fluid Dynamics, Vol. 1, S. Friedlander and D. Serre, eds., North-Holland, Amsterdam, (2002), 71-305. 